\title{
MATHEMATICAL METHODS IN ANCIENT ASTRONOMY
}

\author{
O. NEUGEBAUER
}

\section{Contents}

1. Chronological remarks . . . . . . . . . . . . . . . . . . . . . 1013

2. Introduction .................................... 1014

A. Celestial mechanics

3. Greek astronomy .................................. 1016

4. Babylonian astronomy ........................... 1020

B. Numerical and graphical methods

5. Introduction; the linear methods . . . . . . . . . . . . . . . 1026

6. Spherical trigonometry ............................ 1028

7. The "Analemma" ................................. 1030

8. The "Planisphaerium" ............................... 1034

9. Map projection ................................ 1037

1. Chronological remarks. The main source for our knowledge of ancient astronomy is Ptolemy. His Mathematical composition, commonly known as the Almagest, quotes observations of his own ranging from 127 to 142 A.D. $[35 ; 36 ; 37] .{ }^{1}$ This work seems to be the earliest of a whole series of fundamental works, such as his Geography $[41]$, the Tetrabiblos $[39 ; 40]$, and so on, whose influence on mediaeval thought cannot be overrated.

Questions of historical priority will not be discussed here. Nevertheless it must be emphasized that Ptolemy relied heavily on methods developed by his predecessors, especially Hipparchus. Indeed, almost all our information about the latter's work is based on references in the Almagest. However fragmentary our knowledge of Hipparchus' astronomy may be, it is evident that it represents a milestone in the development of mathematical astronomy. Observations of Hipparchus quoted by Ptolemy extend from 162 to 127 B.C.

Finally, we have original Babylonian ephemerides for the moon and the planets covering, with only minor gaps, the years from 227 to 48 B.C. (Kugler [19;20], Schnabel [47], Schaumberger [20], Neugebauer [31]). Nothing is known about the exact date or origin of the Babylonian methods though it might be a fair guess to assume a date between 400 and 250 B.C.

To our knowledge, Egypt exercised no positive influence on the development of mathematical astronomy. This is in perfect accord

An address delivered before the New York meeting of the Society on April 16, 1948 , by invitation of the Committee to Select Hour Speakers for Eastern Sectional Meetings; received by the editors May 28, 1948.

1 Numbers in brackets refer to the bibliography at the end of the paper. 
with the fact that Egyptian mathematics never went beyond an extremely elementary level, totally unfit for the description of astronomical phenomena.

2. Introduction. It is not the scope of this paper to give even a sketch of the historical development of ancient astronomy. I shall only try to illustrate the close relationship between mathematics and astronomy, a relationship which goes much farther than one might assume at first sight. I shall mention only three problems which seemingly would belong to purely observational astronomy but actually are essentially dependent upon mathematical theories. These problems are (a) the determination of the apparent diameter of the moon, (b) the determination of the constant of precession, (c) the determination of geographical longitude. The first two problems were not solved by direct measurement but by relating them to the theory of the motion of the moon. It requires the whole mechanism of the lunar theory to compute the coordinates of the moon for given eclipses, especially the distance of the center of the moon from the center of the shadow. It is only after these elements were found according to the mathematical theory that the observed magnitudes of the eclipses are used to find the apparent diameter of the moon (Almagest V, 14). Similarly, the longitudes of fixed stars are not measured directly but are referred to the moon, and thus eventually to the sun, by means of occultations or close conjunctions. Again the whole lunar theory is required to find the common longitude of moon and star (Almagest VII, 3). Finally, the determination of geographical longitude is based on the simultaneous observation of a lunar eclipse, the circumstances of which must be determined from theory. This last problem involves, however, another theoretical consideration. The ancients measured time not by means of clocks of uniform rate but by sun dials and waterclocks which showed "seasonal" hours. Seasonal hours can be simply described as an extremal form of "daylight saving time" because each hour is always the 12th part of the actual length of daylight. Thus the time reckoning is fully adjusted to the variation of the seasons. For civil life, this undoubtedly has its great advantages. For astronomical computations, however, the reduction of seasonal hours to equinoctial hours requires a theory of the dependence of sun dials upon the geographical coordinates and the longitude of the sun.

These examples will suffice to make it understandable that two groups of mathematical theory play a paramount role in ancient astronomy. On the one hand, a detailed celestial mechanics is needed, 
especially for the theory of the moon; on the other hand, auxiliary problems must be solved, including the theory of celestial and terrestial coordinates, their transformation from one system into another, and their application to the theory of various types of sun dials. In short, we can say that kinematics and spherical astronomy play a much greater role than empirical observations. The ancient astronomers were fully aware of the fact that the low accuracy of their instruments had to be supplemented by a mathematical theory of the greatest possible refinement. Observations are more qualitative than quantitative: "when angles are equal" may be decided fairly well on an instrument but not "how large are the angles," says Ptolemy with respect to the lunar and solar diameter (Almagest V, 15; Heiberg p. 417). Consequently, period relations over long intervals of time and lunar eclipses are the main foundations so far as empirical material is concerned; all the rest is mathematical theory. We shall see that this holds for Greek as well as for Babylonian astronomy.

The fact that ancient astronomy is to a large extent "mathematics" has far-reaching consequences for the history of civilization. The Middle Ages inherited an astronomical system, and with it a picture of the structure of the universe, of a consistency and inner perfection which hardly seemed open to improvement. The bearers of the Christian civilizations, at the very beginning, had lost contact with Hellenistic science; hence the astronomy of Western and Central Europe relapsed for many centuries into a primitive stage of knowledge where a few simple period relations sufficed as the basis of the computation of Easter and similar problems. Though this process was to some extent delayed by the continued use of astronomical tables for astrological purposes, the destruction of the ancient tradition would have been complete had not Greek astronomy found a new and most interesting development among Hindu astronomers. When the Arab conquest reached India, Greek astronomy soon saw a triumphant revival everywhere in the Moslem world, thus preparing the basis for the new development of astronomy and mathematics in the Renaissance. The "Ptolemaic system" has of ten been blamed for the preservation for almost 1500 years of a narrow, yet much too complicated, picture of the world. ${ }^{2}$ It is only fair to underline the fact that this system pre-

\footnotetext{
2 It should be remarked that "Ptolemaic system" is often used in a rather unhistoric fashion. Actually the Aristotelian version of the homocentric spheres of Eudoxus determined the cosmological ideas of the philosophers and theologians of the Middle Ages. Thus a system 500 years older than the Almagest should take most of the blame.
} 
served for the same length of time the tradition of mathematical methods which became most powerful tools in the hands of Copernicus, Tycho Brahe and Kepler.

\section{A. Celestial mechanics}

3. Greek astronomy. The cornerstone of Greek celestial mechanics is an essentially "dynamical" principle, however metaphysical its actual formulation in Greek philosophical literature may sound. It is the idea that the circular movement of celestial bodies is the only movement which can last eternally. This "principle of inertia" is the guiding principle of all Greek astronomical theories. The fortunate accident that the orbits in our planetary system deviate very little from circles made it possible to construct geometric models whose gradual improvement corresponds to the addition of new Fourier terms, each of which has a certain physical significance. Modern scientists have of ten declared simplicity to be the criterium of truth, and a whole philosophy of "economy of reasoning" has offered its guidance to the researcher. This school of thought could rightly claim the early Greek astronomers as its first followers. Nothing "simpler" and more natural could have been assumed than the preference of celestial bodies for circular movements. And the remarkable successes of this assumption could only strengthen confidence in its correctness. Nobody could foresee that the simplicity of the circular movements is due to an accidental distribution of masses or that this also causes the simplicity of Newton's law, conveniently hiding from us the effects of a general gravitational space.

Here is not the place to describe the development of astronomical hypotheses, based on the combination of circular movements, or, originally, of movements of spheres, following an ingenious idea of Eudoxus. This development is described in masterly fashion by Duhem in his Système du monde [18]. We shall here restrict ourselves to a short discussion of some points in the theory of eccenters and epicycles in its application to the lunar movement. For the theory of Mercury and Venus see Boelk [3] and Schumacher [48].

Assuming that all circular movements proceed at constant angular velocity, it is obvious that an observer who is not located at the center gets the impression of a variable velocity. We know that already Apollonius, about 200 B.C., knew that an eccentric movement can be replaced by an epicyclic movement, where the center of the epicycle moves on the deferent with the mean angular motion around the observer whereas the object moves on the circumference of the epicycle with the same angular velocity in the opposite direction. 
The radius of the epicycle is identical with the eccentricity of the eccenter. Similar relations hold for more general cases and it is therefore a matter of choice which hypothesis is used in a specific case (Almagest XII, 1 and III, 3). We know, for example, that Hipparchus used an epicycle for the description of the solar anomaly (Theon Smyrnaei, De astronomia, XXXIV [50]) while Ptolemy preferred the eccenter because of its greater simplicity, using only one motion (Almagest III, 4).

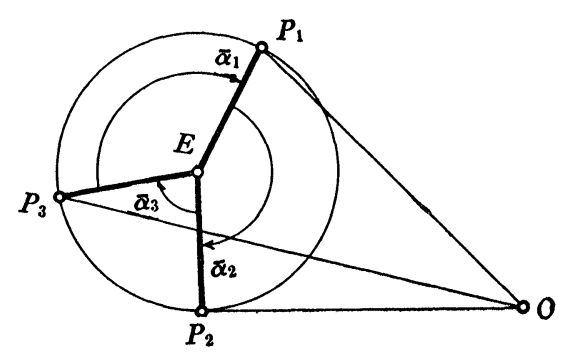

FIG. 1

The determination of the parameters of models of this type requires great ingenuity. As an example I might quote the case of the lunar theory in its simpler (Hipparchian) form which only accounts for the "first anomaly," that is, the eccentricity with uniformly progressing apsidal line. To this end an epicyclic model is assumed and three lunar eclipses are observed, giving the true longitudes $\lambda_{1}, \lambda_{2}, \lambda_{3}$ at given moments $t_{1}, t_{2}, t_{3}$. The mean motions in mean longitude $(\bar{\lambda})$ and mean anomaly $(\alpha)$ are known from period relations. Thus it is possible to find the positions of the moon on its epicycle, expressed by the differences of their anomalies (cf. Fig. 1). Furthermore the corresponding mean longitudes $\bar{\lambda}_{i}$ can be found for each $t_{i}$. The differences $c_{i}=\lambda_{i}-\bar{\lambda}_{i}$ are the corresponding values of the equation of center and can be interpreted as the angles under which the radii $E P_{i}$ appear from the observer (Almagest IV, 6). The solution of this problem, undoubtedly known to Hipparchus and discussed in detail by Ptolemy, is often needed in surveying; one then speaks of the "Pothenot" or "Snellius" problem (Tropfke [51, V, 97]; the identity of these problems has been seen by Delambre [4, II, 164] and Oudemans [33]). As a result the radius of the epicycle can be found in terms of the radius of the deferent. Thus the eccentricity of the lunar orbit is known.

Before we proceed to Ptolemy's addition to Hipparchus' theory of 
the moon we might for a moment discuss the often underlined contrast between the simplicity of Kepler's theory and the clumsiness of eccenters and epicycles. Actually one compares here theories of different level. If one accepts the statement that the earth or the moon travels on an ellipse, one disregards perturbations and describes the true longitude in first approximation by

$$
\lambda=\bar{\lambda}+2 e \sin \alpha,
$$

where $\alpha$ is the mean anomaly. But exactly the same relation holds for an eccenter, or the equivalent epicycle, if its eccentricity is $2 e$. If we want to take the movement of the apsidal line into account, again both models are equivalent because a uniform rotation of the apsidal line must be added in both cases. The error of the ancient theory does not at all lie in its unnecessary complication but in its simplicity, which leads only to correct longitudes whereas the distances are very badly represented, especially near the apogee. Because the observation of distances requires the measurement of very small angular differences for parallax or apparent diameters, this part of the theory was very difficult to check. Consequently one distrusted the observation of small angles even in cases where one should have found the discrepancy and preferred a simpler model to added corrections. For the longitudes, however, the ancient theory is exactly as simple and as efficient as Kepler's theory within the same first order approximations.

As was mentioned in the preceding remarks, the fundamental parameters of the lunar theory were obtained from eclipses, thus for syzygies. Already Hipparchus started to test the theory also for intermediate quadratures, and observations of his, quoted by Ptolemy (especially Almagest V, 5; Heiberg p. 369), showed that the "mean apogee" of the epicycle, from which $\alpha$ has to be counted, cannot be considered fixed. Ptolemy further investigated this situation and constructed a model which coincides with the simple theory in the syzygies and shows the proper fluctuation of the longitudes in the quadratures, depending on mean anomaly $\alpha$ and double mean elongation $2 \epsilon$. The corresponding term $a \cdot \sin (2 \epsilon-\alpha)$ in the modern theory is called "evection." Because the value of the constant $a$ is about $1 ; 16^{\circ}$ whereas $2 \epsilon=6 ; 17^{\circ}$, we have for the syzygies and quadratures respectively ${ }^{3}$

$$
\lambda=\bar{\lambda}+6 ; 17^{\circ} \sin \alpha \mp 1 ; 16^{\circ} \sin \alpha
$$

which shows that the maximum of the equation of center varies be-

8 I use the notation $a, b ; c$ for $a \cdot 60+b+c \cdot 60^{-1}$. 
tween about $5 ; 1^{\circ}$ and $7 ; 33^{\circ}$. The Ptolemaic values are $5 ; 1^{\circ}$ and $7 ; 39^{\circ}$ respectively (Almagest $\mathrm{IV}, 10$, and $\mathrm{V}, 8$ ). Also for intermediate elongations and anomalies Ptolemy's representation of the longi-

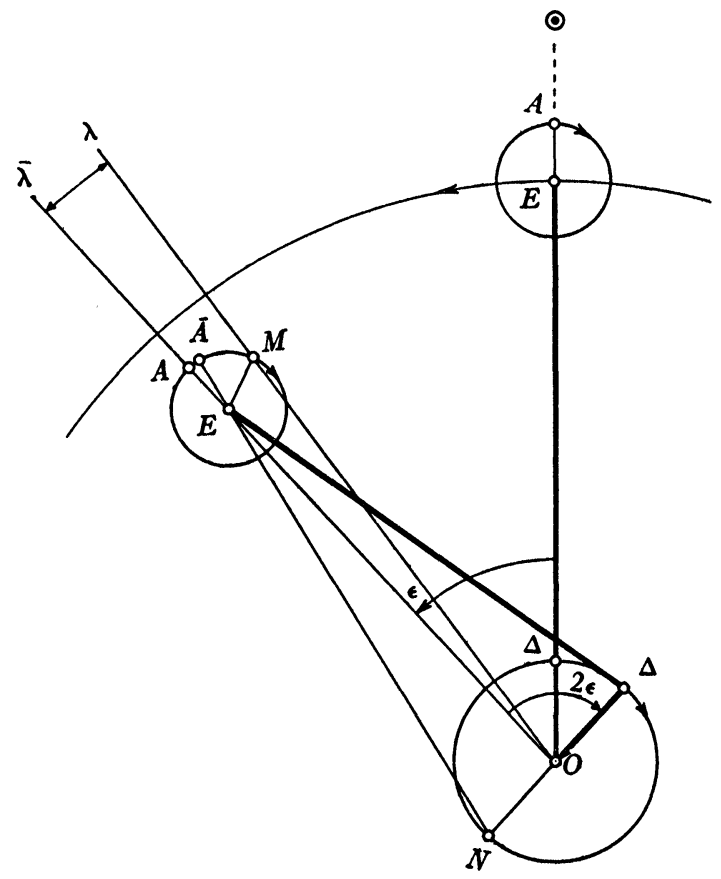

FIG. 2

tudes is very good (Kempf [15]). His model is described by Fig. 2 . In order to obtain the necessary increase of the equation of center from conjunction towards quadrature, he moves the lunar epicycle closer to the observer $O$, by letting $\Delta$ travel on a circle around $O$ such that $\Delta$ has the elongation $2 \epsilon$ from the mean moon $E$. Simultaneously the oscillations of the anomaly are obtained by introducing a "mean apogee" $\bar{A}$ from which $\alpha$ is to be counted. The position of $\bar{A}$ is defined by means of the rule that $\bar{A} E$ always points towards the point $N$ which is diametrically opposite to $\Delta$ on the circle with center $O$.

If we want to compare this model with a geometric representation of the modern theory, using the same degree of approximation, we have to represent the evection as follows (Möbius [24]). The center $C$ of the elliptic orbit rotates around the mean center $\bar{C}$ on a circle of 
radius $\bar{e} / 5$ (where $O \bar{C}=\bar{e}$ ) with an angular velocity which is twice the velocity of the elongation of the mean sun from the mean apsidal line of the lunar orbit. Hence not only the apsidal line but also the eccentricity varies according to position and length of $O C$. Translating these movements into the language of eccenters and epicycles one obtains a model which closely resembles Ptolemy's construction, though with the essential difference that the radius of the epicycle must be made a function of $\alpha$. Again one sees that Ptolemy's model is not too complicated but too simple because it represents only the longitudes correctly at the expense of the distances.

4. Babylonian astronomy. The final goal of Greek and Babylonian astronomy is, of course, identical. Starting from a few empirical elements one wishes to be able to compute the positions of the celestial bodies for any given moment. One may say with equal right that the progress, or the error, of the Greek method consisted in the invention of an intermediate step, namely the construction of a "dynamical" model, based on circular movements. From this model the Greeks derived their numerical tables exactly as a modern "Nautical Almanac" is derived from computations which are determined by consequences of dynamical rules and certain empirically determined initial values. As far as we can say from the material available to us, it seems that no such theoretical model existed in Babylonian astronomy. One apparently tried to obtain, on purely mathematical grounds, the rules for the computation of the tables from the empirical data. To use modern terminology the Babylonian procedure is very close to harmonic analysis. Given periodic phenomena of a rather complex character; find simple periodic functions, whose combination describes, within given limits of accuracy, the observed phenomena. While in our harmonic analysis the basic periodic functions admit, at least in principle, a direct geometric interpretation by simple harmonic oscillations, no such interpretation is obvious for the functions used in Babylonian astronomy. We do not know what were the Babylonian concepts about the physical structure of the universe. It seems safe to say that whatever concepts might have existed they were not directly reflected in the mathematical methods for the lunar and planetary movements.

The fundamental tools of Babylonian astronomy are periodic difference sequences of first and higher order. The simplest case is represented by tables in which each line represents a certain moment, these moments being equidistant, for example, one mean synodic month apart. The tabulated values can therefore be represented as a function $f(n)$ for integer values of the argument. Linear interpolation 
then leads to a continuous function $f(n)$ whose graph looks like Fig. 3. We shall call such functions "linear zigzag functions." The period of the function $f(x)$ may be called $P$ and it is obvious that

$$
P=2 \Delta / d
$$

where $\Delta$ is the amplitude and $d$ the slope of the increasing branch.

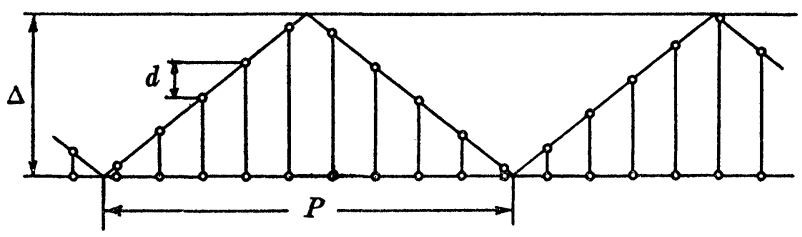

FIG. 3

The tabulated values $f(n)$ have, of course, in general a much larger period than $P$ because $P$ is in practice never an integer. If

$$
P=2 \Delta / d=\pi / Z
$$

where $\pi$ and $Z$ are relative prime integers, we see that $\pi=P Z$ is the smallest integer after which the sequence $f(n)$ repeats all its values. We thus call $\pi$ the "number period," $Z$ the "wave number," that is, the number of waves of length $P$ contained in $\pi$. Ordinarily $\pi$ is a large number. If we, for example, know that one year contains $12 ; 22,8$ mean synodic months we have $12 ; 22,8=46,23 / 3,45$ which shows that $\pi=46,23=2783$ and $Z=3,45=225$. This means that " 2783 mean synodic months $=225$ years" is the smallest period relation on which a zigzag function with $P=12 ; 22,8$ is built.

Functions of this type are especially used in the lunar theory. Two coexisting "systems" are known. One, called "System B," uses for the variable solar velocity a linear zigzag function; "System A," however, assumes a constant solar velocity for an arc of the ecliptic, with a discontinuous change to another value, constant on the remaining arc. The assumption of system $B$ seems much more natural than the very bold assumption of sudden jumps in the solar velocity. Actually, however, the theory of system B is much more involved in its purely mathematical consequences. The reason can be outlined as follows. Let $f(t)$ be a periodic function of $t$, for example, the latitude of the moon, represented by a linear zigzag function of period $p_{0}$. An "ephemeris" is a table of values of $f(t)$ for equidistant values of $t$, for example, for all mean conjunctions. If the solar velocity varies from a value $w$ to $W$ then the lunar velocity must also vary from 
$360+w$ to $360+W$ in order to maintain the same mean distance between conjunctions. On the other hand the anomaly of the sun must not influence the mean distance between consecutive nodes. Developing the consequences of this requirement one finds that the tabulated values of $f(t)$ again form a linear zigzag function but with difference $d$ where the solar velocity is $w$, and with difference $D$ where the solar velocity is $W$. It then holds that

$$
D-d=\frac{\Delta}{3,0 p_{0}} \cdot \frac{Y}{Y+1}(W-w)
$$

where $\Delta$ is the amplitude of $f(t)$ and $Y$ the length of the solar year. This formula shows immediately the essential difference between the systems A and B. If the solar velocity is restricted to two values only, fixed in their relation to the ecliptic (system A), then two differences suffice for the computation of $f(t)$. If, however, the solar velocities form a linear zigzag function (system $B$ ) then the same holds for the differences of $f(t)$ and $f(t)$ is a difference sequence of second order (Neugebauer [27]). Because we are actually dealing in these tables with arithmetical functions, instead of continuous functions, it is not at all trivial to determine the parameters of the zigzag function $g(n)$ of the differences of $f(n)$. The solution of this problem is found by considering mean periods and mean differences, taken over a whole number period. The mean value $\mu_{g}$ of $g$ is then defined by

$$
\mu_{o}=2 \Delta \frac{1-p_{0}}{p_{0}}=d_{0}
$$

where $d_{0}$ is the mean slope of $f(n)$. Arithmetical problems of this type were obviously the reason for assuming in system $\mathrm{A}$ a discontinuous change in the solar velocity at two points only instead of adopting the model of system B with its much greater mathematical complications.

In dealing with difference sequences of second order, Babylonian astronomers came close to problems whose importance was again seen only in the early days of the development of calculus. As an example may be quoted two sequences in system $\mathrm{B}$, called $H$ and $J$, whose astronomical significance is here without interest. $H$ is a linear zigzag function with minimum 0 and maximum $\Delta_{H}$. The values of $H$ are the differences of $J$. The mean value of $J$ is 0 and its amplitude is determined in such a way that increasing branches change to decreasing branches and vice versa whenever $H$ is zero. In other words $H$ behaves like the derivative of $J$. Fig. 4 shows in the lower part the function $H$, in the upper part the function $J$. I have chosen an example where an error has occurred at the decreasing branch of the 
second wave of $J$. The correct value (dotted line) would have been negative. Instead, the computer took the positive sign and continued thereafter according to the rule. Our graph shows how strongly the shape of the curve is affected by this error. On the other hand, it underlines the agreement between the extrema in $J$ and the zeros in
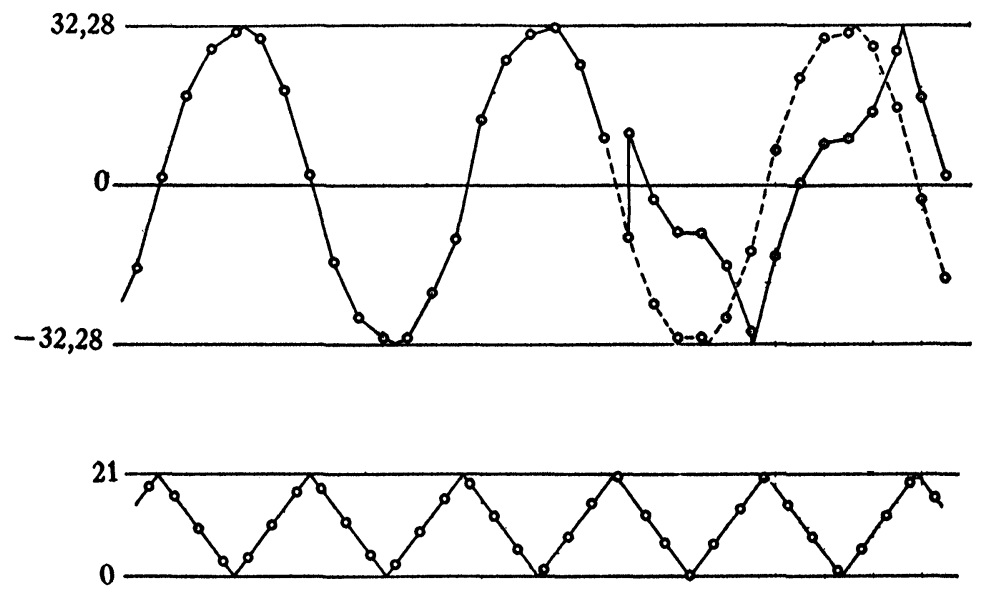

FIG. 4

$H$ in the normal case though we are dealing only with discrete values and not with continuous functions. The question arises how one could determine the amplitude $\Delta_{J}$ of a sequence of discrete values to a given function $H$ such that nevertheless a behavior of this type was achieved. The answer can be given as follows. We simply require that the relation between $H$ and $J$ be correct "in the mean" over the whole number period $\pi$ of $H$. In an interval of length $\pi$ the function $J$ must oscillate, for example, from maximum to maximum $Z / 2$ times because two waves of $H$ correspond to one oscillation of $J$. The total change of values of $J$, caused by $H$, is the total of all differences, thus $\sum H=\mu \pi$ where $\mu$ is the mean value of $H$. This total change for a single wave of $J$ is $2 \Delta_{J}$. Thus we have

$$
\begin{gathered}
2 \Delta_{J} \cdot Z / 2=\mu \pi \quad \text { or } \\
\Delta_{J}=\mu \pi / Z .
\end{gathered}
$$

This is actually the relation which is satisfied by the parameters of the sequences found in lunar ephemerides.

Many examples could be quoted for the extensive use which Babylonian astronomers made of difference sequences to represent periodic functions. We have examples of modified zigzag functions. The main part increases or decreases linearly. In order to avoid, however, the sharp change of direction at the extrema, sequences of 
second order which bridge the neighborhood of the extrema are constructed.

The idea of operating with interpolations obviously influenced the whole procedure of describing planetary movements. Apparently no attempt was made to find the longitude $\lambda$ of a planet directly as function of $t$. The following very clever indirect method was invented. Instead of dealing with the total motion, single "phenomena" were considered as if they were independent celestial bodies. In the case of Mercury, to mention a specific example, four phenomena are considered: first and last appearance as morning star, and first and

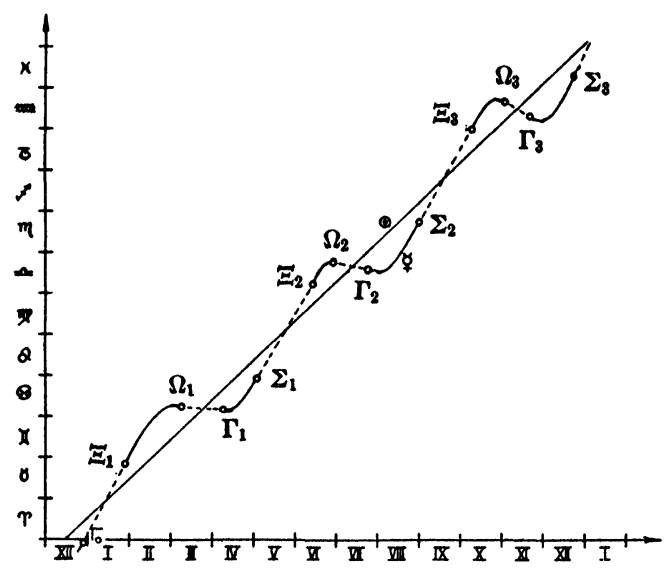

FIG. 5

last appearance as evening star. Now, for example, the first appearance as evening star is taken independently, and rules are given to compute tables for the dates and longitudes of this single phenomenon. Here again, there exist different "systems" for the description of the dependence of these coordinates on the zodiac, that is, implicitly on the longitude of the sun. We have cases of step functions or linear zigzag functions, obviously several systems being used for the same planet. After the coordinates of one phenomenon were found for a period of time, the coordinates for the remaining phenomena were computed in a similar way, though generally with different functions, for the same period. The result is a complete list of all phenomena which now could also be read in their natural order. Finally, interpolation is used for the coordinates of the planet for intermediate dates.

This whole procedure can best be illustrated in an example for 
Mercury (cf. Fig. 5). In the first step all points $\boldsymbol{\Xi}_{i}$ were computed. Three different zones of the zodiac are distinguished such that the distances between consecutive points $\Xi$ depend upon the zone to which they belong. Different zones determine the distribution of the points $\Gamma_{i}$. Eventually, the points $\Omega_{i}$ and $\Sigma_{i}$ can be found from the corresponding points $\Xi_{i}$ and $\Gamma_{i}$ respectively. Thus we end up with a sequence of irregularly distributed points near the straight line which represents the movement of the sun. The variability of the distances between these points causes also variable differences between consecutive points for different phenomena, for example, $\Omega$ and $\Gamma$. Because the planet is retrograde between $\Omega$ and $\Gamma$, this indicates different velocities of retrogradation. In order to find these velocities, schemes of interpolations are used. Fig. 6 shows an example; the

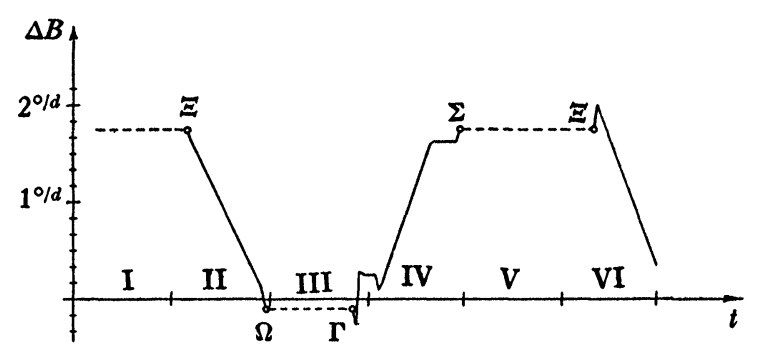

FIG. 6

retrograde motion is represented by the constant velocity $-0 ; 6$ degrees per day. Another section of constant velocity $\left(+1 ; 45^{\circ / d}\right)$ is assumed from $\Sigma$ to $\Xi$. In both sections the planet is near the sun and therefore invisible. For the other parts of the orbit, linearly variable differences are used (except for small irregularities near the end points, probably due to the practical requirement of relatively round numbers for the interpolation). As a result, day-to-day positions (Fig. 7) which represent a very satisfactory representation of the actual movement are obtained. It is clear that these methods require the knowledge of the summation formulae of arithmetic progressions.

These examples suffice to characterize the principle methods of Babylonian astronomy. For details of the planetary theory one may consult Kugler [20], Pannekoek [34], van der Waerden [52; 53]. The historical influence of these arithmetical methods is very great. They opened, for the first time, the way for a consistent numerical treatment of astronomical phenomena. This influence is still felt today in the use of sexagesimal units in the measurement of time and 
angles. In antiquity this influence was much more strongly visible in many cases which have left no trace in the modern development. Ptolemy's table of refraction, for instance, assumes that the angles of the reflected ray form a second degree sequence as function of the angle of the incident ray (Lejeune [21]). Babylonian methods are predominant in astronomical computations for astrological purposes

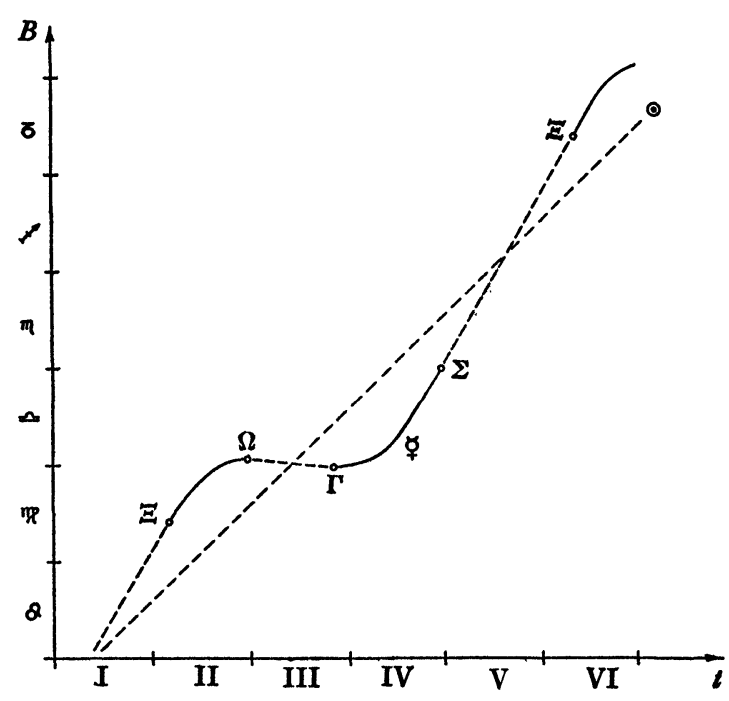

FIg. 7

for the obvious reason that they are easier to handle and do not require full understanding of complicated geometrical models. Greek papyri (Knudtzon-Neugebauer [17], to be supplemented by P. Ryl. 27 [14]) show the use of Babylonian period relations. Ancient and mediaeval geography was deeply influenced by concepts based on Babylonian methods. We shall return to these "linear methods" in the next section.

\section{B. NUMERICAL AND GRAPHICAL METHODS}

5. Introduction; the linear methods. The fundamental role of the eccenters and epicycles for Greek astronomy and the dramatic history of the discussion of these geometric assumptions in the Renaissance have left us with the impression that Greek astronomy was mainly geometrical in character. Though it is evident that the Greek astronomers must have felt that their models somehow reflected physical facts, at least in theory the geometric models were only the 
tools for the computation of tables. Consequently we find many tables in the Almagest whose construction involved an enormous amount of numerical work. According to the contents they fall naturally into tables for spherical astronomy (chords, rising times, zenith distances, and so on), tables for the sun (mean motion, equation of center), for the moon (mean motion for longitude, anomaly, argument of latitude, anomaly, equation of center, evection, parallax, mean conjunctions, eclipses) and for the planets (mean motion, anomaly, stationary points, elongation, latitudes, heliacal risings and settings).

The Babylonian influence on Greek numerical methods is obvious from the use of the sexagesimal division of all units. Unfortunately this influence did not fully penetrate all computational steps. Thus of ten we find fractions expressed by means of unit fractions, replacing, for example, $0 ; 42$ by $1 / 2+1 / 5$. Though this notation never occurs in tables, it influenced the accuracy of single calculations because frequently results are rounded off in order to obtain simple unit fractions. Thus, for example, Ptolemy finds in Almagest IV, 6 the value $5 ; 13$ or $5 ; 14$ for the radius of the lunar epicycle (Heiberg [35, pp. 313 and 322$])$ but he later always uses $51 / 4(=5 ; 15)$ as a round value. In general it must be said that the ancients were little concerned about the influence of rounding off and accumulated errors. Often the errors are of the same order of magnitude as the effect under consideration. Apparently it was only under the influence of modern analysis that we have learned to consider the evaluation of errors as an essential part of numerical methods. On the other hand it must be said that, for example, excellent approximations of square roots were developed very early. The Babylonians of the second millenium B.C. already used alternating geometric and harmonic means (Neugebauer $[26$, p. $33 \mathrm{ff}$.$] ) and many values in the Almagest$ can be explained by this method. It would not be surprising if this technique reached the Greeks together with the sexagesimal system and it might be significant that the value $2^{1 / 2}=1 ; 21,50,10$ is not only found in cuneiform records (Neugebauer-Sachs [32, p. 32]) but is used by Ptolemy in the computation of the chord of $90^{\circ}$ (Heiberg $[35$, p. 35,15$])$.

The arithmetical methods of the Babylonian astronomers are also discernible in another field of ancient astronomy. We have already mentioned the "seasonal hours" for ancient time measurement which thus requires a knowledge of the law of variation in the length of daylight whenever astronomical computation with equinoctial hours was needed. For the Babylonian astronomers the length of daylight 
was of great importance also because one of the main goals of their lunar theory was the prediction of the evening of first visibility of the new crescent of the moon after conjunction. This evening defined the beginning of a new month and consequently the whole calendar depended on this problem. For its solution the knowledge of the moment of sunset is, of course, required. Because the length of daylight is the time from sunrise to sunset, one also may ask for the time it takes the semicircle of the ecliptic from $\lambda$ to $\lambda+180$ to rise if $\lambda$ is the longitude of the sun at the given moment. A month later the longitude of the sun will be given roughly by $\lambda+s$ where $s=30^{\circ}$ is the length of one zodiacal sign. Now the length of daylight is the rising time of the semicircle from $\lambda+s$ to $\lambda+s+180$. Its value can be obtained from the previous value by adding the rising time of the zodiacal sign $s+180$ and by subtracting the rising time of $s$. Hence we see that the length of daylight can be found if the rising times of ecliptic arcs are known. The rising time of a given arc of the ecliptic depends obviously on its variable inclination to the horizon. To obtain an insight into the relationship between rising times, seasons and geographical location can be called the central problems of early Greek spherical astronomy. Its complete solution is found in the tables of the Almagest where the rising times are given for every 10 degrees for all latitudes whose longest daylight varies between $12^{\mathrm{h}}$ and $17^{\mathrm{h}}$ in steps of $1 / 2^{\mathrm{h}}$.

Ptolemy already makes full use of spherical trigonometry. The Babylonian astronomers, however, used also here arithmetical schemes to describe the values of the rising times of the zodiacal signs as function of the longitude. Two methods were developed: a crude approximation related to system A of the solar theory, and a more refined scheme in system B. Both are built on arithmetical progressions. The Greeks expanded these methods by varying the parameters linearly, thus introducing geographical zones of given length of daylight, known as "climates." This concept remained fundamental for ancient and mediaeval mathematical astronomy (Honigmann [13], Neugebauer [29]). Ptolemy himself uses the rising times of system A in the Tetrabiblos ([40, I, 20 p. 94-95]; [39, I, 21 p. 46]) and thus contributed to securing the survival of Babylonian methods for many centuries.

6. Spherical trigonometry. The lack of a convenient algebraic notation prevented the Greeks from condensing the solution of a general triangle into a single formula instead of solving two right triangles. Of greater consequence was their use of chords in a circle of radius 60 instead of the trigonometric functions. Consequently their plane 
trigonometry is expressed in the relations

$$
\begin{aligned}
a & =\frac{c}{120} \operatorname{crd}(2 \alpha), \\
b & =\frac{c}{120} \operatorname{crd}(180-2 \alpha), \\
\frac{a}{b} & =\frac{\operatorname{crd}(2 \alpha)}{\operatorname{crd}(180-2 \alpha)}, \\
c^{2} & =a^{2}+b^{2} .
\end{aligned}
$$

Tables for crd $\alpha$ are given, for example, in Almagest I, 11 in steps of $1 / 2^{\circ}$ and with an accuracy of two sexagesimal places. Their computation is based on the so-called Ptolemaic theorem for a quadrilateral and its diagonals inscribed in a circle.

As plane trigonometry is described by the four above relations (1) to (4), so spherical trigonometry contains four similar relations which we may describe in our symbols by

$$
\begin{aligned}
\sin \alpha & =f(a, c), \\
\cos \alpha & =g(b, c), \\
\tan \alpha & =h(a, b), \\
\cos c & =\cos a \cos b .
\end{aligned}
$$

Menelaus, about 100 A.D., already knew that a spherical triangle is determined by its angles (Spherics I, 18 Krause [18, p. 138]). Yet relations of the type

$$
\begin{aligned}
& \cos a=\phi(\alpha, \beta), \\
& \cos c=\psi(\alpha, \beta)
\end{aligned}
$$

do not seem to have been discovered before the Arabs.

In general, it is my impression that spherical trigonometry was completed rather late in the development of Greek science. Its problems and methods were exclusively determined by astronomical needs and astronomical concepts. This is obvious in the earliest treatises, about 300 B.C., by Autolycus [2] and Euclid [9], but it also holds for Theodosius ([49], Schmidt [46]), who probably was a younger contemporary of Hipparchus (Ziegler [56]). The astronomical importance of parallel circles certainly contributed to obscuring for a long time the necessity of restricting oneself to great circles, a discovery which very well may have been Menelaus' great contribution. Even Ptolemy did not yet have a clear concept of the 
possibility of replacing spherical triangles by plane triangles. In the computation of the components of parallax in Almagest V, 19 (Heiberg [35, p. $456 \mathrm{f}$.$] ), he treats a right spherical triangle with one$ very small angle, but with two large sides, as if it were a plane triangle in order to find the second angle at the small base, though this is just the case where also the second angle is very close to $90^{\circ}$. It has often been conjectured that already Hipparchus was able to solve problems of spherical trigonometry. This seems rather implausible in view of the above-stated facts, and indeed we shall see (p. 1036) that there existed methods to avoid completely spherical trigonometry. Hence I see no reason for assuming that the central theorem of ancient and Arabic spherical trigonometry, the "Menelaus theorem," was known before Menelaus. That its two forms are not independent was remarked by Theon ([43, p. 569], Rome [44]).

7. The "Analemma." At least since the early part of the third century B.C., astronomers were able to predict solar and lunar positions with a high degree of accuracy. In particular, the longitude of the sun could be considered as known for any given date. Ecliptic coordinates, however, are not directly visible in the sky. The daily rotation moves all celestial objects around the pole of the equator, and thus relates the measurement of time to equatorial coordinates. Yet local noon is again determined by the sun and thus, by the relation of ecliptic coordinates to the local coordinates of the observer, horizon and meridian. All these coordinates play a role in the practical measurement of time by means of sun dials, the simplest form of which is the vertical "gnomon" on a horizontal plane. Finally, different observers had to establish their relative positions through the determination of their geographical coordinates. It is therefore no great wonder that we can observe that ancient astronomers concentrated a great deal of attention on the theory of spherical coordinates in their relation to celestial and terrestrial objects and the theory of sun dials.

Ptolemy's role in this branch of astronomy can be well appreciated because we have not only a work of his own, called the Analemma ([38, p. $187 \mathrm{ff}$.$] , Luckey [22]) but we also know a little about his$ predecessors. We can see that Ptolemy had all the essential methods, inherited from earlier times, but loaded down with historical relics which made their application unnecessarily clumsy. Ptolemy rationalized the whole procedure, down to the smallest details. First of all, he introduced coordinates whose mutual relations are independent of the geographical position. We consider the octant of the celestial 
sphere which contains the sun $\Sigma$, and whose vertices are the zenith $Z$, South $S$, and East $E$ (cf. Fig. 8 left). From each of these points we draw a great circle to the sun, calling the respective arcs "descensivus" (=zenith distance), "horarius" and "hectemoros." Each pair of these arcs may be used to determine the position of the sun.
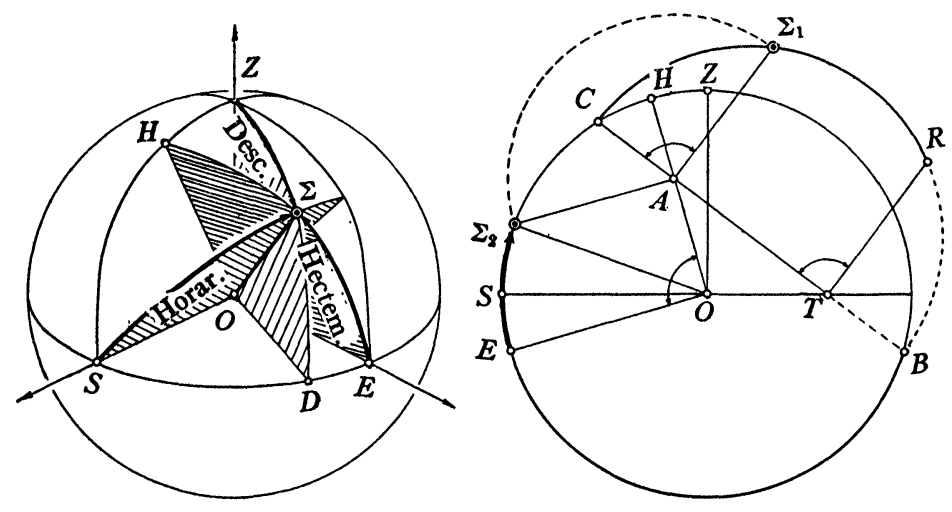

FIG. 8

This perfectly symmetric arrangement can be compared with the older system. There the position of the sun was given by the horarius and the arc of the equator from $E$ to the plane of the horarius. Only for the equinoxes does this arc end at the sun (then being identical with the hectemoros), but ordinarily it meets the horarius in a point which is not only different from the sun but which also depends on the geographical latitude of the observer. Another pair of "old" coordinates was descensivus and "antiskion," that is, the azimuth of the direction of the shadow counted from $S$ to $D$.

Ptolemy now treats in great detail the methods to determine his coordinates for a given solar position. The right part of Fig. 8 describes his solution for the hectemoros. Let the circle around $O$ be the plane of the meridian. The geographical latitude of the observer determines the inclination of the equator, and from the solar tables we can find the longitude of the sun for the given moment. As we shall see presently, this also determines the position of the path of the sun, henceforth called its "daily circle." Its intersection with the plane of the meridian may be $C B$. Ptolemy now proceeds in the typical fashion of "descriptive geometry." He revolves the plane of the daily path of the sun about its trace $C B$ into the plane of the meridian. $C$ is the culminating point; vertically above $A$, we have the given position $\Sigma_{1}$ of the sun which rose at $R$, the arc $B R$ being below 
the horizon. Because $A$ is the orthogonal projection of the sun onto the plane of the meridian, we know that $A$ belongs also to the plane of the hectemoros. Because also $O$ belongs to both the plane of the meridian and the plane of the hectemoros we have in $O A H$ the trace of the plane of the hectemoros in the plane of the meridian. Perpendicular to this trace is the line $O E$ where $E$ represents the East point of the horizon, revolved about $O H$ into the plane of the meridian. The distance $A \Sigma_{1}$ is a true distance. Using it as a radius we find on the celestial sphere $\Sigma_{2}$, which gives the position of the sun in the plane of the hectemoros, turned into the plane of the meridian. Thus the arc $E \Sigma_{2}$ is the true "hectemoros" we wanted to find.

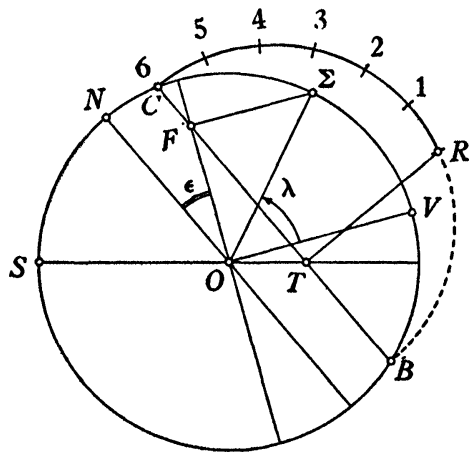

FIG. 9

A similar procedure can be followed for the other angles. The method consists, in general, in constructing first the Cartesian coordinates of the sun with respect to the fundamental planes of the octant $Z S E$ and then revolving the Cartesian coordinate planes about the coordinate lines in order to get the angles in true size.

It follows from the preceding discussion that the whole construction of all Ptolemaic coordinates requires only the main circle with center $O$ and the parallel circle whose diameter $C B$ is determined by the time of the year, that is, by the longitude of the sun. Ptolemy thus proceeds to construct a nomogram whose central part is the great circle with center $O$. Added are half circles of the type $B C$ corresponding to the solar path from month to month. This system of circles is mounted on a turntable with various scales for the reading of the angles. This makes it possible to bring the equator in the proper position towards a given horizon. Rectangular plates placed on the proper points of the scales then allow the direct determination 
of the angles required (cf. for details and for a figure Luckey [22]). A mechanized age can only be delighted with the extremal efficiency of this apparatus, of which nothing has come down to us except for an incomplete description in a Greek palimpsest and a poor Latin translation by William of Moerbeke.

Ptolemy is not the inventor of descriptive geometry. More than a century before his time, Vitruvius describes in his De architectura the Analemma in connection with the construction of sun dials ([54]). From Vitruvius we also learn how to determine graphically the path of the sun for a given longitude $\lambda$. Let the circle around $O$ again be the meridian (Fig. 9); then the trace $O N$ of the equator is given for a given place. If $\epsilon$ denotes the obliquity of the ecliptic, we also know the trace $O F$ of the ecliptic. Revolving the plane of the ecliptic into the plane of the meridian we find the vernal point $V$ on the end point of the radius $O V$ perpendicular to $F O$. Hence $\Sigma$ is the sun for given $\lambda$, and $F B$ the trace of its path. If we divide $R C$ into six equal parts we have the position of the sun for each seasonal hour from sunrise $(R)$ to noon $(C)$.

The theory of sun dials leads to the solution of two problems in the history of mathematics, problems whose fame is inversely proportional to their interest: the date of Heron of Alexandria and the origin of the conic sections.

It is in itself of very little importance to establish accurately the date of a rather mediocre author whose role in the history of science is due only to the fact that so much else is lost. Yet it is somewhat unsatisfactory to know no more about an of ten quoted writer than that he lived sometime between -200 and +300 . It is therefore a pleasant side result of the study of an analemma, described by Heron in his Dioptra ([12], Rome [42], Neugebauer [28]), to see that the elements which he quotes for a lunar eclipse fit exactly one and only one eclipse between -200 and +300 , namely the partial eclipse (magnitude 8 digits) of A.D. 62 March 13. It is extremely plausible to assume that the writing of the Dioptra fell close to the occurrence of this eclipse. This is indeed the only excuse for selecting an example whose date coincides almost with equinox, because the main problem in the analemma discussed by Heron consists in reducing local seasonal time of one place (Rome) to the local seasonal time of another locality (Alexandria); this problem loses its importance only twice a year, namely at the equinoxes, when seasonal time and equinoctial time coincide. Only the desire to quote a real eclipse, which had occurred recently, can have led to quoting as an example a date which fell only a few days before equinox. If we thus must 
place Heron into the end of the first century A.D. we support at the same time an argument of J. Klein [16, p. 135, note] who conjectured that Heron and Diophantus were contemporary, both belonging to the time of Nero.

For us the interest in Heron's analemma consists in the direct relationship it establishes between astronomy and mathematical geography. The only method in antiquity to determine the geographical longitude of a place consisted in using lunar eclipses as time signals. The comparison of two local seasonal times is made by Heron by means of a hemispherical sun dial combined with an analemma of the Vitruvian type. Its main idea is to use the analemma for each place and thus to find the position of the local meridian with respect to the sun. Transferring the results into the hemispherical dial gives directly the angular difference between the meridians. This is one of several examples which show the intimate combination of geometrical construction and the direct use of globes or hemispheres in Greek astronomy. In general it may be said that Greek mathematics is often much less "pure" than is generally assumed.

This latter remark might be kept in mind when we try to relate one of the most interesting subjects of Greek geometry, the conic sections, to an astronomical origin. It is well known that these curves were defined by Apollonius (about 200 B.C.) as the intersection of a circular cone by planes of variable inclination, whereas the "old" geometers considered only right circular cones, intersected by a fixed plane perpendicular to one generating line. The different types of these curves were obtained by varying the angle at the vertex (cf., for example, Heath [1]). This definition suggests immediately that its origin is to be sought in the fixed right angle between the gnomon and the receiving plane of the shadow. Indeed, we have only to point the gnomon towards the culminating point to obtain exactly the configuration required (Neugebauer [30]). The sun travels on its "daily circle," its rays form the right circular cone, whose vertex is the tip of the gnomon. At noon the gnomon falls into one generating line and the receiving plane is perpendicular to it. The shadow describes a hyperbola. The natural question, namely, how these curves depend on the declination, is equivalent to asking how the angle at the vertex of the cone influences the shape of the curve. This is exactly the form in which the conic sections were studied by Menaechmus (about 350 B.C.).

8. The "Planisphaerium." The importance of spherical astronomy is reflected in the manifold of mathematical tools developed in order 
to solve its main problems. Eventually spherical trigonometry superseded all other methods which were, in all probability, invented before spherical geometry was sufficiently far advanced to reach numerical results. At that early stage the methods of descriptive geometry, reflected in the Analemma, might seem the most natural way to transform arcs on the sphere into arcs of one plane. Another work of

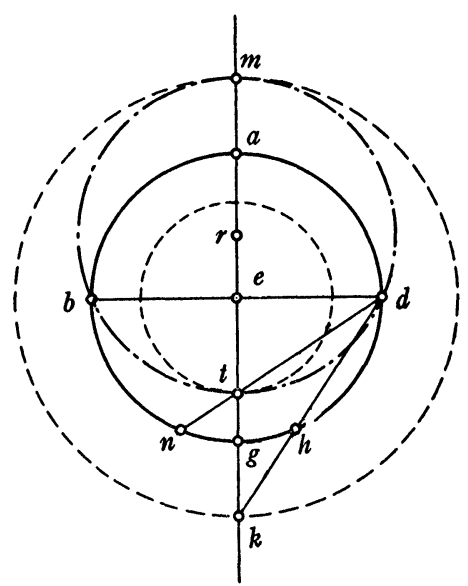

FIG. 10

Ptolemy, the Planisphaerium ([38, p. 227-259]; Drecker [5]), shows, however, that also stereographic projection was known, in particular its important quality of mapping circles into circles, straight lines included. No proof is given for this fact by Ptolemy, a clear indication that he is operating on well known grounds, developed long before his time.

The projection chosen maps the whole sphere onto the plane of the equator with the south pole as center of projection. In order to determine the center and the radius of the image of a circle, descriptive methods are again employed. As a simple example might be quoted the determination of the solstitial circles and of the ecliptic. Let abgd be the equator (Fig. 10); we then consider the same circle also as a picture of the meridian, turned about the diameter ag into the plane of the equator. Consequently $d$ represents the south pole. Let $g h=g n$ $=\epsilon=23 ; 51^{\circ}$. The point $h$ is therefore a point of the diameter of the winter solstitial circle, $n$ of the summer solstitial circle. Their projections from $d$ are $k$ and $t$ respectively. Because all parallel circles have their center in $e$, the images of both circles are found. Because the ecliptic must be represented by a circle touching the solstitial circles 
in $t$ and $m$ respectively, also this circle is known. It is then proved that the straight line $b d, b$ and $d$ being the equinoxes, passes through $e$, a general relation which is of ten used in the following for intersecting great circles. The conformity of the mapping was apparently unknown.

The main goal of the whole procedure is the determination of the rising times for the zodiacal signs. In order to find the representation of these signs one has only to construct the parallel circles of given declination by the same method which was used for the solstitial circles. The declinations of given ecliptic points are considered known, but it is in principle important to remark that they can also be found by geometric construction, namely from the "daily circle" in the analemma (cf. above p. 1031). Finally it is easy to construct the circle which represents the horizon for a given latitude. The variable positions of the ecliptic with respect to the horizon at different times of the year are in our projection represented by different positions of the horizon circle with respect to the fixed image of the equatorecliptic system. In order to find the rising times of a given arc of the ecliptic we have only to construct the two positions of the horizon passing through its end points. These two horizon circles intersect the equator in two points whose angular distance is the rising time in question. Because angles on the equator are represented without distortion, our problem is solved by this construction.

The above description shows that the "planisphaerium" could be used for a purely graphical or mechanical solution of problems of spherical astronomy. This was indeed the use made of this method especially by the Arabs whose "astrolabes" are based on the projections described here (Drecker [6] and [7], Michel [23]). In Ptolemy's treatise, however, a different attitude is taken. The geometric constructions are only used for transforming spherical problems into problems of plane geometry which then are solved numerically by means of plane trigonometry. Obviously we have here before us the method used before spherical trigonometry was invented, that is, before the Menelaus theorem was known.

Much speaks in favor of the assumption that the planisphaerium was the tool of Hipparchus (Delambre [4, II p. $453 \mathrm{ff}$.], Drecker [6, p. $16 \mathrm{ff}]$.$) . All computations are based on the latitude of Rhodes,$ where Hipparchus made his observations. Synesius of Cyrene ascribes the invention of the "astrolabe" to Hipparchus (FitzGerald [10, p. $263]$ ); this statement is certainly to be taken seriously in view of the fact that Synesius was a pupil of Hypathia, who collaborated with her father Theon on the commentaries to the Almagest (Rome [43, 
p. LXXXIII]). Finally, Hipparchus determines the positions of stars by a combination of ecliptic and equator coordinates (Vogt [55]); he takes the longitude of the point where the circle of declination through the star meets the ecliptic and then uses the remaining declination as second coordinate. This system finds its direct explanation in the planisphaerium: the first coordinate is given on the image of the ecliptic whereas the circles of declination are mapped into radii.

9. Map projection. The work of Mžik and Hopfner [25] has given us a good understanding of Greek mathematical geography as con-

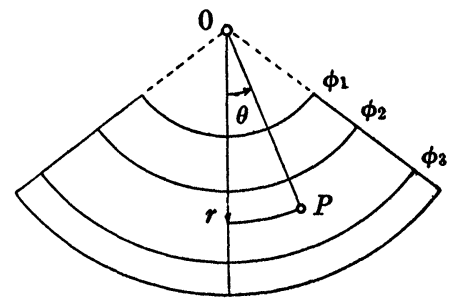

FIG. 11

tained in the first book of Ptolemy's Geography. I shall give a short summary, using modern terminology, which will suffice to show how far the Greeks had come in the general problem of mapping a sphere onto a plane. From the earlier development we only know that Ptolemy's predecessor, Marinus (about 100 A.D.), used a cylinder projection which can be described by

$$
y=\phi, \quad x=\lambda \cos 36^{\circ}
$$

where $x$ and $y$ are the Cartesian coordinates of the map, $\phi$ the geographical latitude, $\lambda$ the geographical longitude, and $\phi=36^{\circ}$ the latitude of Rhodes. Obviously this projection preserves latitudes on all meridians and longitudes for the parallel of Rhodes.

Ptolemy introduced two types of conic projection. In the first type he maps meridians on radii, parallels of latitude on circles with center $O$ (Fig. 11). In order to determine the parameters of this mapping three conditions are imposed. We introduce polar coordinates $r$ and $\theta$, and call $\phi=90-\phi$ the colatitude.

Condition 1: preservation of length on all meridians

$$
r=\phi+c \text {. }
$$

Condition 2: preservation of length for the latitude $\phi_{2}=36^{\circ}$ of 
Rhodes. Thus

$$
r\left(\Phi_{2}\right) \cdot \theta=\sin \phi_{2} \cdot \lambda
$$

or from (1)

$$
\theta=\frac{\sin \bar{\phi}_{2}}{\bar{\phi}_{2}+c} \cdot \lambda .
$$

Condition 3: preservation of the ratio of lengths on the parallels

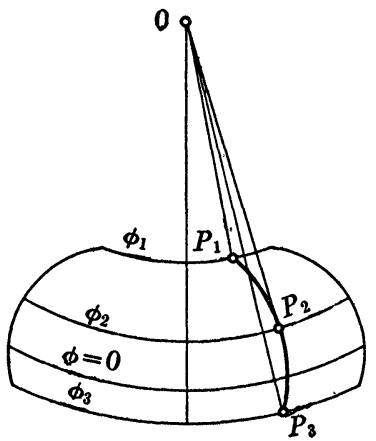

FIG. 12

of Thule $\left(\phi_{1}=63\right)$ and on the equator $\left(\phi_{3}=0\right)$. Hence $c$ can be determined from

$$
\frac{\sin \Phi_{1}}{\sin \bar{\phi}_{3}}=\frac{\Phi_{1}+c}{\bar{\phi}_{3}+c} .
$$

It is obvious that this projection is an improvement of Marinus' simple cylinder projection.

The second type of conic projection, proposed by Ptolemy, assumes that both meridians and parallels of latitude are represented by circles. We again use polar coordinates (Fig. 12) but only the circles $r=$ const. now have geographical significance as images of circles of constant latitude. The radii, however, are no longer meridians. Nevertheless it is required that length is preserved on all radii. Thus we have

$$
r=\bar{\phi}+c_{0}
$$

where $c_{0}$ is an arbitrary parameter which gives the value $r=r_{0}$ of the image of the equator. In order to determine the circles which represent the meridians we need three points. Thus we can impose 
the following three conditions: preservation of length on each of the following three parallels $\phi_{1}=63$ (Thule), $\phi_{2}=\epsilon=23 ; 50$ (Syene in Egypt), and $\phi_{3}=-16 ; 25(\phi=16 ; 25$ is the latitude of Meroe on the Upper Nile). Thus

$$
r\left(\bar{\phi}_{i}\right) \cdot \theta=\sin \bar{\phi}_{i} .
$$

For a given value of $\lambda$ we can find three values of $\theta$ by substituting in (5) the values $\bar{\phi}_{1}=27, \bar{\phi}_{2}=66 ; 10 \quad \phi_{3}=106 ; 25$. The values $r\left(\phi_{i}\right)$ are similarly given by (4); thus we are able to construct the circle $P_{1} P_{2} P_{3}$ which represents the meridian $\lambda$. If (5) were assumed to hold for all latitudes, the meridians would no longer be circles but we would obtain the Bonne projection, which is preserving length in radial direction and on all parallels.

In concluding, I want to repeat that the topics discussed here were not intended to give a complete picture of mathematical problems which originated from problems of astronomical character. Nevertheless, they may suffice as an illustration of the close relationship between mathematics and astronomy in antiquity, a relationship which thereafter never lost its importance. "Astronomy proceeds to its demonstrations in no uncertain way, for it uses as its servants geometry and arithmetic, which it would not be improper to call a fixed standard of truth" (Synesius of Cyrene in his letter to Paeonius, about 400 A.D.; FitzGerald [10, p. 262]; cf. Almagest I, 1; Heiberg $[1$, p. $6,17 \mathrm{ff}]$.$) .$

\section{BIBLIOGRAPHY}

1. Apollonius of Perga, Treatise on conic sections, ed. in modern notation by T. L. Heath, Cambridge University Press, 1896.

2. Autolyci de sphera quae movetur liber, de ortibus et occasibus libri duo, ed. Fr. Hultsch, Leipzig, Teubner, 1885.

3. Paul Boelk, Darstellung und Priffung der Mercurtheorie des Claudius Ptolemaeus, Thesis, Halle, 1911.

4. Delambre, Histoire de l'astronomie ancienne, Paris, 1817.

5. J. Drecker, Das Planisphaerium des Claudius Ptolemaeus, Isis vol. 9 (1927) pp. 255-278.

6. - Des Johannes Philoponus Schrift ïber das Astrolab, Isis vol. 11 (1928) pp. 15-44. 219.

7. - Hermannus Contractus, Über das Astrolab, Isis, vol. 16 (1931) pp. 200-

8. Pierre Duhem, Le système du monde, histoire des doctrines cosmologiques de Platon a Copernic, 5 vols., Paris, Hermann, 1913-1917.

9. Euclidis opera omnia, vol. 8, Phaenomena et scripta musica, ed. H. Menge, Leipzig, Teubner, 1916.

10. Augustine FitzGerald, The letters of Synesius of Cyrene, Oxford Universitv Press, 1926. 
11. Heiberg, see Ptolemaeus [35].

12. Herons von Alexandria, Vermessungslehre und Dioptra, Griechisch und deutsch von Hermann Schöne, Leipzig, Teubner, 1903.

13. Ernst Honigmann, Die sieben Klimata, Heidelberg, Winter, 1929.

14. Arthur S. Hunt, Catalogue of the Greek papyri in the John Rylands Library in Manchester, vol. I, Literary texts (Nos. 1-61), Manchester, 1911.

15. Paul Kempf, Untersuchungen über die Ptolemäische Theorie der Mondbewegung, Thesis, Berlin, 1878.

16. Jacob Klein, Die griechische Logistik und die Entstehung der Algebra. II, Quellen und Studien z. Geschichte der Mathematik B vol. 3 (1934) pp. 122-235.

17. Erik J. Knudtzon and $\mathrm{O}$. Neugebauer, Zwei astronomische Texte, K. humanistiska Vetenskapssamfundets i Lund Årsber. 1946-1947 II, pp. 77-88.

18. Max Krause, Die Sphärik von Menelaos aus Alexandrien in der Verbesserung von $A b \bar{u}$ Nașr Manșür $b$. 'Ali $b$. 'Irāq, Abhandlungen Gesellschaft der Wiss. zu Göttingen, Philol.-hist. K1., 3. Folge, vol. 17, 1936, Berlin, Weidmann.

19. Franz Xaver Kugler, Die babylonische Mondrechnung, Freiburg, Herder, 1900.

20. - Sternkunde und Sterndienst in Babel, Münster, Aschendorff, 2 vols., 1907-1924; 3 Ergänzungshefte 1913-1935 (No. 3 by Johann Schaumberger).

21. Albert Lejeune, Les tables de réfraction de Ptolémée, Annales de la Société scientifique de Bruxelles (1) vol. 60 (1946) pp. 93-101.

22. P. Luckey, Das Analemma von Ptolemäus, Astron. Nachrichten vol. 230 (1937) cols. 17-46.

23. Henri Michel, Traité de l'astrolabe, Paris, Gauthier-Villars, 1947.

24. August Ferdinand Möbius, Gesammelte Werke, Leipzig, Hirzel, 1887.

25. Hans v. Mżik and Friedrich Hopfner, Des Klaudios Ptolemaios Einfïhrung in die darstellende Erdkunde, Klotho vol. 5 (1938).

26. O. Neugebauer, Vorgriechische Mathematik, Berlin, Springer, 1934.

27. - Untersuchungen zur antiken Astronomie, III, Quellen und Studien zur Geschichte der Mathematik, B vol. 4 (1937) pp. 193-346.

28. —— Über eine Methode zur Distanzbestimmung Alexandria-Rom bei Heron, Kgl. Danske Videnskabernes Selskab, Historisk-Filologiske Meddelelser vol. 26, 2 (1938) and vol. 26, 7 (1939).

29. - On some astronomical papyri and related problems of ancient geography. Transactions of the American Philosophical Society, n.s. vol. 32, 2 (1942) pp. 251-263.

30. - The astronomical origin of the theory of conic sections, Proceedings of the American Philosophical Society vol. 92 (1948) pp. 136-138.

31. - Astronomical cuneiform texts, in preparation.

32. O. Neugebauer and A. Sachs, Mathematical cuneiform texts, American Oriental Series, vol. 29, New Haven, 1945.

33. J. A. C. Oudemans, Lösung des sog. Pothenotschen, besser Snellius'schen Problems von Ptolemaeus, Vierteljahrsschrift d. Astronomischen Gesellschaft. vol. 22 (1887) pp. 345-349.

34. A. Pannekoek, Calculation of dates in the Babylonian tables of planets, Akademie van Wetenschappen te Amsterdam, Proceedings vol. 19 (1916) pp. 684-703.

35. Claudii Ptolemaei Opera quae extant omnia, vol. I, Syntaxis mathematica, ed. J. L. Heiberg, Leipzig, Teubner, 1898, 1903.

36. Des Claudius Ptolemäus Handbuch der Astronomie, aus dem Griechischen übersetzt von Karl Manitius, Leipzig, Teubner, 1912, 1913.

37. Composition mathématique de Claude Ptolémée, trad. par M. Halma et suivie des notes de of M. Delambre, Paris, 1813, 1816 [reprinted Paris, Hermann, 1927]. 
38. Claudii Ptolemaei opera quae extant omnia, vol. II, Opera astronomica minora, ed. J. L. Heiberg, Leipzig, Teubner, 1907.

39. Claudii Ptolemaei opera quae extant omnia, vol. III, 1 Apotelesmatica, ed. F. Boll-Ae. Boer, Leipzig, Teubner, 1940.

40. Ptolemy, Tetrabiblos, transl. by F. E. Robbins, The Loeb Classical Library, 1940.

41. Claudii Ptolemaei geographia, ed. C. F. A. Nobbe, Leipzig, 1843.

42. A. Rome, Le probleme de la distance entre deux villes dans la Dioptra de Héron Annales de la Société scientifique de Bruxelles vol. 42 (1922-1923) Mémoires pp. 234-258.

43. - Commentaires de Pappus et de Théon d'Alexandrie sur l'Almageste, Roma and Città del Vaticano, 1931, 1936, 1943 (Studi e Testi 54, 72, 106).

44. - Les explications de Théon d'Alexandrie sur le théorème de Ménélas, Annales de la Société scientifique de Bruxelles, ser. A, vol. 53 (1933) CR pp. 39-50.

45. Schaumberger, see Kugler [20].

46. Olaf Henric Schmidt, Studies on ancient sphaeric, Thesis, Brown University, 1942 .

47. Paul Schnabel, Berossos und die babylonisch-hellenistische Literatur, Leipzig, Teubner, 1923.

48. C. J. Schumacher, Untersuchungen ïber die ptolemäische Theorie der unteren Planeten (Merkur und Venus), Münster, Aschendorff, 1917.

49. Theodosius Tripolites, Sphaerica, ed. J. L. Heiberg, Abhandlung der Gesellschaft der Wissenschaften zu Göttingen, Philologisch-Historische Klasse, NF 19, 3 (1927), Berlin, Weidmann.

50. Theonis Smyrnaei Platonici liber de astronomia, ed. Th. H. Martin, Paris, 1849.

51. Johannes Tropfke, Geschichte der Elementar-Mathematik, vols. 1 to 4 in 3d ed., 1930-1940, vols. 5 to 7 in 2d ed., 1923, 1924, Berlin-Leipzig, De Gruyter.

52. B. L. van der Waerden, Zur babylonischen Planetenrechnung, Eudemus vol. 1 (1941) pp. 23-48.

53. - Egyptian "Eternal Tables," Nederlandsch Akademie van Wetenschappen, Proceedings vol. 50 (1947) pp. 536-547, 782-788.

54. Vitruvius, On Architecture, transl. by Frank Granger, The Loeb Classical Library, 1945.

55. H. Vogt, Versuch einer Wiederherstellung von Hipparchs Fixsternverzeichnis, Astronomische Nachrichten vol. 224 (1925) cols. 17-54.

56. Konrat Ziegler, Theodosius, Paulys Real-Encyclopädie der classischen Altertumswissenschaften, vol. 5 A, cols. 1930-1935.

BROWN UNIVERSITY 